\section{Qualidade da dieta e despesa diária com alimentação em adultos com doença cardiovascular no Brasil}

\author{
Quality of diet and daily spending on food by \\ adults with cardiovascular disease in Brazil
}

\section{Calidad de la dieta y gasto diario en alimentación de adultos con enfermedad cardiovascular en Brasil}

Camila Ragne Torreglosa 1,2

Flávia Mori Sarti 1

Ângela Cristine Bersch-Ferreira 2 Bernardete Weber 2

Renato Hideo Nakagawa Santos 2

Alexandre D. P. Chiavegatto Filho 1

doi: 10.1590/0102-311X00225019

\section{Resumo}

A adesão a uma alimentação saudável depende de fatores como os preços dos alimentos, sendo que alguns estudos conduzidos em países desenvolvidos apontam para um maior custo de uma alimentação de melhor qualidade nutricional. O objetivo do presente trabalho foi avaliar o custo direto da alimentação de indivíduos adultos com doença cardiovascular no Brasil, investigando a relação entre o custo e a qualidade da dieta. Foram utilizados os dados de um ensaio clínico randomizado, o BALANCE Program. A investigação atual é uma análise transversal no momento inicial dos participantes com alta adesão ao estudo realizado em 35 centros das cinco regiões brasileiras. O consumo alimentar de amostra com 1.160 indivíduos foi coletado pelo recordatório alimentar de 24 horas (R24h), a avaliação da qualidade da dieta pelo Índice da Qualidade da Dieta Revisado $(I Q D-R)$ e os custos diretos da alimentação foram estimados por meio de preços de mercado. Não foram observadas diferenças significativas no custo direto da alimentação ou características dos indivíduos entre os tercis de adesão. Quando analisados todos os recordatórios não houve correlação entre custo e qualidade da dieta $(r=0,38 ; p=0,17)$, já a análise por tercis mostrou fraca correlação entre o menor tercil de adesão ( $r$ $=-0,112 ; p=0,03)$. O presente estudo apontou ausência de diferenças entre os custos diretos da alimentação classificada como saudável e daquela com a pior qualidade nutricional, o que pode ser um incentivo à adesão às orientações alimentares no Brasil, minimizando barreiras à adoção de estilos de vida saudáveis.

Prevenção Secundária; Dieta; Custos e Análise de Custo
Correspondência

C. R. Torreglosa

Hospital do Coração.

Rua Abrão Dib 4, São Paulo, SP 04004-030, Brasil.

camilatorreglosa@gmail.com

1 Universidade de São Paulo, São Paulo, Brasil.

2 Hospital do Coração, São Paulo, Brasil. 


\section{Introdução}

As doenças cardiovasculares (DCV) são hoje uma das mais importantes causas de óbito no mundo 1, sendo responsáveis por cerca de $30 \%$ dos óbitos no Brasil 2 . O infarto agudo do miocárdio e o acidente vascular cerebral são as principais DCV no país ${ }^{2}$. A modificação do estilo de vida traz um importante benefício para a prevenção da DCV, uma vez que boa parte dos fatores de risco é considerada modificável 3. A prevenção bem-sucedida em longo prazo requer a adoção de um estilo de vida saudável baseado na cessação do tabagismo e etilismo, prática regular de atividade física e adoção de uma dieta saudável 1,4,5,6,7.

No caso da alimentação, há vários fatores de influência sobre as decisões de consumo alimentar, incluindo sabor, praticidade e preços dos alimentos, destacando-se a influência do custo da alimentação na adoção de determinados tipos de alimentos componentes de um padrão alimentar saudável 8 .

O custo da dieta está associado a diversos fatores que vão desde a aquisição dos produtos alimentícios até os gastos de tempo e outros recursos necessários ao preparo dos alimentos. Alguns estudos recentes apontam associação direta entre qualidade nutricional e custo da alimentação 9 . Uma revisão da literatura concluiu que alimentos de baixo valor nutricional apresentam menor preço por caloria e tendem a ser escolhidos no momento da compra, principalmente pelos indivíduos de baixa renda 9. Certamente, esse hábito não favorece o equilíbrio alimentar. Um estudo realizado com dados de famílias brasileiras não foi capaz de predizer uma alimentação que atingisse a recomendação de nutrientes sem aumento no custo ${ }^{10}$. Apesar disso, uma análise de preços por grupo alimentar isolado, ainda com famílias brasileiras, aponta o inverso, sendo favorável ao aumento do consumo de alimentos com melhor qualidade alimentar por serem mais acessíveis 11

Assim, tendo em vista a possibilidade de influência do custo da alimentação sobre a adesão a uma alimentação saudável e ainda com lacuna para a discussão na população brasileira, o presente trabalho analisou o custo direto da alimentação entre indivíduos adultos com DCV, assim como a sua relação com a qualidade nutricional da dieta.

\section{Métodos}

\section{Desenho do estudo}

Análise transversal de uma subamostra do ensaio clínico randomizado multicêntrico em nível nacional, que avaliou os efeitos de uma alimentação cardioprotetora entre indivíduos em acompanhamento para a prevenção secundária de DCV no Brasil entre 2012 e 2017, denominado BALANCE Program (Bringing Active Living and Nutrition into your Childhood Everyday).

\section{Amostra}

Dados de 2.534 adultos com evidência de aterosclerose manifesta (doença arterial coronariana, doença cerebrovascular ou doença arterial periférica), recrutados em 35 centros de pesquisas nas cinco macrorregiões brasileiras e acompanhados ao longo de até quatro anos, foram utilizados para a estimativa de custos e qualidade nutricional da alimentação. O estudo foi aprovado pelo Comitê de Ética em Pesquisa do Hospital do Coração de São Paulo e os participantes assinaram termo de consentimento livre e esclarecido no início da pesquisa 12.

A análise dos custos diretos e qualidade nutricional da dieta foi conduzida em uma subamostra dos participantes da pesquisa que apresentaram alta adesão ao protocolo. Assim, foram incluídos neste trabalho somente os dados do momento inicial de indivíduos com presença em mais de $80 \%$ das consultas individuais, que atenderam aos telefonemas de seguimento do estudo principal. A amostra final consistiu de 1.160 indivíduos (40\% da amostra original). 


\section{Variáveis}

Informações sobre as características sociodemográficas e econômicas (sexo, idade, escolaridade, região de residência e posse de bens) e indicadores de saúde (tipo de DCV: doença arterial coronariana, doença cerebrovascular ou doença arterial periférica) dos participantes foram coletadas pessoalmente por pesquisadores treinados.

A classificação socioeconômica foi realizada pela definição da Associação Brasileira de Empresas de Pesquisa (Critério de Classificação Econômica Brasil. http://www.abep.org/criterio-brasil, acessado 04/Jan/2020), que classifica o indivíduo em oito classes (A1, A2, B1, B2, C1, C2, D e E). Neste estudo, as classes foram reduzidas para: A, B, C, D e E. A escolaridade foi classificada em: analfabeto (nenhum estudo), Ensino Fundamental 1 (completo ou incompleto), Ensino Fundamental 2 (completo ou incompleto), Ensino Médio (completo ou incompleto) e Ensino Superior (completo ou incompleto).

Dados de consumo alimentar foram coletados em dois momentos (tempos $=0$ e 15 dias) antes da intervenção do estudo principal por meio da aplicação de recordatório alimentar de 24 horas (R24h), instrumento cuja aplicação envolve o relato dos alimentos e bebidas consumidos no dia anterior à realização da entrevista. O registro eletrônico do R24h e a análise de composição nutricional da alimentação relatada foram realizados por meio de digitação dos dados no sistema NutriQuanti (http:// vivandapesquisa.com.br/).

\section{Estimativa de custos diretos da alimentação}

Os custos diretos da alimentação foram calculados usando-se a coleta de dados de preços de mercado para alimentos e preparações relatados nos R24h dos indivíduos participantes da pesquisa, no período entre março de 2013 a abril de 2015 (consultas inicias e de 15 dias do estudo), contemplando sazonalidade na alimentação populacional com base na distribuição das entrevistas realizadas ao longo do ano em cada região do país. Foram registrados 1.103 alimentos e preparações em uma lista compilada após a exclusão de itens repetidos.

O levantamento dos preços dos alimentos e ingredientes das receitas foi realizado em três supermercados (lojas físicas) localizados no Nordeste (Fortaleza, Ceará), Centro-oeste (Goiânia, Goiás) e Sul (Pelotas, Rio Grande do Sul) do Brasil, assim como em duas cadeias de supermercados nacionais (lojas on-line) entre outubro e dezembro de 2018.

O período da coleta de dados de preços foi definido após a aprovação de extensão do projeto de pesquisa para a estimativa de custos do programa. Assim, o processo de atualização monetária dos preços para a estimativa de custos da alimentação de cada indivíduo considerou deflatores específicos segundo tipo de alimento, local de residência e data de coleta dos dados de R24h, utilizando dados oficiais de inflação do Índice de Preço ao Consumidor Amplo (IPCA), disponibilizados pelo Instituto Brasileiro de Geografia e Estatística (IBGE. https://www.ibge.gov.br/estatisticas/economicas/precose-custos/9256-indice-nacional-de-precos-ao-consumidor-amplo.html? =\&t=o-que-e, acessado em 04/Jan/2020).

O painel de números-índice de cada produto consumido pelos indivíduos em cada período e em cada região foi usado para a composição da base de dados dos deflatores específicos (15.120 deflatores), a fim de refletir adequadamente os preços relativos dos alimentos adquiridos pelos participantes no momento da entrevista, contemplando características culturais locais e sazonalidade, seguido de atualização dos valores de dezembro de 2018.

A equipe de coleta de dados de preços foi especificamente treinada para o levantamento de informações de preço, tipo de embalagem e peso bruto do alimento, baseando-se em formulário específico para a coleta de dados de preços de mercado em equipamentos de varejo. O registro de preços de alimentos sempre foi baseado em coleta de informações sobre o maior número de produtos disponíveis do mesmo tipo de alimento no mesmo estabelecimento, sendo incluídas informações de até três alimentos (por exemplo, pelo menos três marcas diferentes do mesmo item, incluindo o menor e o maior preço). Ao final da coleta de dados no varejo, foi empregada a média do preço dos alimentos como preço final no estabelecimento pesquisado. $\mathrm{O}$ mesmo procedimento foi repetido nos cinco estabelecimentos de varejo pesquisados de forma padronizada, em qualquer região do país. 
O preço médio de cada alimento foi convertido em preço por grama do produto pronto para consumo, utilizando-se o padrão de fatores de correção e cocção e ingredientes descritos nos livros de receitas do BALANCE Program e na tabela para avaliação de consumo alimentar em medidas caseiras ${ }^{13}$. À exceção dos ingredientes principais, cuja quantidade foi estimada valendo-se do relato de cada participante, as quantidades de temperos das preparações foram padronizadas em $5 \%$ de açúcar, $1 \%$ de sal, $2 \%$ de óleo de soja ou 10\% de óleo de soja em frituras.

Finalmente, o custo direto global de cada dia alimentar relatado pelos participantes no período do estudo foi estimado pela multiplicação do preço por grama dos itens alimentares pela quantidade consumida. O custo direto final de cada dia alimentar foi atualizado e convertido em dólares americanos (USD), utilizando-se a taxa de câmbio oficial publicada pelo Banco Central do Brasil em dezembro de 2018 ( 1 USD = 3,88 BRL), data de referência para a atualização dos dados monetários no presente trabalho.

\section{Avaliação da qualidade nutricional da dieta}

O Índice da Qualidade da Dieta Revisado (IQD-R) 14 avalia a qualidade nutricional da alimentação por meio de pontuação do consumo alimentar relatado em relação à adequação no contexto das recomendações nutricionais do Guia Alimentar para a População Brasileira 2006, da Organização Mundial da Saúde, do Instituto de Medicina dos Estados Unidos e das diretrizes da Sociedade Brasileira de Cardiologia. Foi criado e adaptado para a população brasileira com base no Healthy Eating Index 200515.

A pontuação do IQD-R é gerada para cada dia alimentar relatado pelo indivíduo, obtida pela soma de pontos referentes a 12 componentes: nove componentes criados baseando-se nas quantidades consumidas de determinados grupos alimentares (cereais totais, grãos integrais, frutas totais, frutas inteiras, vegetais, legumes e leguminosas verde-escuras e laranja, leite e laticínios, carnes, ovos, leguminosas e óleos), dois componentes baseados na ingestão de quantidade de nutrientes (de gordura saturada e sódio) dentro de determinados limites, e um componente resultante da soma do consumo energético de álcool, açúcar e gordura sólida 14.

Cada R24h coletado junto aos participantes da pesquisa BALANCE Program foi convertido em pontuação de qualidade nutricional segundo o método proposto no cálculo do IQD-R, resultando em uma pontuação que varia entre zero (pior) e 100 pontos (melhor).

\section{Análise estatística}

As variáveis contínuas com distribuição normal foram descritas em termos de média e desvio padrão e as variáveis categóricas em número e percentual.

As associações entre as características sociodemográficas, econômicas e de saúde dos indivíduos (sexo, classe socioeconômica, escolaridade, região de moradia e o histórico de DCV) e os tercis de qualidade nutricional da dieta (calculada pelo IQD-R) foram analisadas por meio de teste qui-quadrado e, no caso da idade, pela análise de variância (ANOVA). A comparação do custo direto da dieta (em USD/dia) entre tercis de qualidade nutricional foi baseada em aplicação de modelo linear generalizado com distribuição gama. Por fim, foi calculada a correlação de Pearson entre IQD-R e custo direto da alimentação em cada categoria de adesão à dieta.

As análises foram realizadas no software R versão 3.6.1 (http://www.r-project.org), considerando-se significância de $5 \%$.

\section{Resultados}

A doença arterial coronariana foi a DCV com a maior frequência entre os participantes do estudo. A maioria dos participantes era homem (59,5\%), com idade média de 62,9 anos, sendo que mais da metade deles tinham menos de 5 anos de escolaridade e renda entre USD 483 e USD 2.414 (classe C) por mês (Tabela 1). Não foram observadas diferenças entre as características dos indivíduos (sexo, idade, DCV e socioeconômicas, escolaridade, classe social e região de moradia) e os escores de adesão ao índice de qualidade da alimentação. O custo direto médio da alimentação dos participantes no 


\section{Tabela 1}

Características dos indivíduos com doença cardiovascular (DCV) participantes do estudo BALANCE Program. Brasil, 2013-2015.

\begin{tabular}{|c|c|c|}
\hline \multirow{2}{*}{$\begin{array}{l}\text { Características } \\
\text { Idade (média, DP) * }\end{array}$} & \multicolumn{2}{|c|}{ Amostra $(n=1.161)$} \\
\hline & 62,9 & 8,0 \\
\hline \multicolumn{3}{|l|}{$\operatorname{Sexo}(n, \%)$} \\
\hline Feminino & 470 & 40,5 \\
\hline Masculino & 691 & 59,5 \\
\hline \multicolumn{3}{|l|}{ Classe socioeconômica (n, \%) } \\
\hline A & 41 & 3,6 \\
\hline B & 327 & 28,8 \\
\hline $\mathrm{C}$ & 648 & 57,1 \\
\hline De E & 119 & 10,5 \\
\hline \multicolumn{3}{|l|}{ Escolaridade (n, \%) } \\
\hline Analfabeto & 290 & 25,6 \\
\hline Ensino Fundamental 1 & 379 & 33,4 \\
\hline Ensino Fundamental 2 & 147 & 13,0 \\
\hline Ensino Médio & 215 & 18,9 \\
\hline Ensino Superior & 104 & 9,2 \\
\hline \multicolumn{3}{|l|}{ Região de moradia no Brasil (n, \%) } \\
\hline Nordeste & 257 & 22,2 \\
\hline Norte & 79 & 6,8 \\
\hline Centro-oeste & 67 & 5,8 \\
\hline Sudeste & 433 & 37,4 \\
\hline Sul & 322 & 27,8 \\
\hline \multicolumn{3}{|l|}{$\operatorname{DCV}(n, \%)$} \\
\hline Doença arterial periférica & 106 & 8,1 \\
\hline Doença arterial coronariana & 1.089 & 83,4 \\
\hline Doença cerebrovascular & 110 & 8,4 \\
\hline
\end{tabular}

DP: desvio padrão.

* Dados referentes ao início do programa.

início do estudo e antes da intervenção foi de USD 3,89 por dia por pessoa, e não foram identificadas diferenças no custo direto da alimentação entre os tercis de adesão (Tabela 2).

Foi testada a correlação entre adesão à dieta de maior qualidade (maior IQD-R) e o custo da alimentação, sendo feito um teste de correlação para a totalidade dos recordatórios coletados $(\mathrm{n}=1.870)$ (Figura 1a) e outros para cada tercil de adesão à dieta de maior qualidade (Figuras 1b, 1c e 1d). Em qualquer um dos cenários, a correlação identificada foi baixa.

\section{Discussão e conclusão}

O artigo analisou a associação entre a qualidade nutricional e o custo direto da alimentação de brasileiros com DCV ( $\mathrm{n}=1.160)$, em uma amostra de 35 centros de pesquisas das cinco regiões brasileiras. A qualidade da alimentação não apresentou associação com o custo direto e também não houve diferença entre o custo direto e os grupos de indivíduos classificados com relação aos escores de adesão ao IQD-R. Os resultados do estudo contribuem à discussão quanto ao efeito dos preços dos alimentos na decisão de compra e consumo de dietas saudáveis, especialmente considerando indivíduos em um país em desenvolvimento.

A comparabilidade dos resultados obtidos em relação às evidências identificadas na literatura é bastante complexa, em decorrência da diversidade de métodos empregados para a análise da qualidade da dieta, assim como em termos de comparação de preços de alimentos e custos das dietas entre 


\section{Tabela 2}

Associação entre escores do Índice de Qualidade da Dieta Revisado (IQD-R) e características de indivíduos e custo direto da alimentação de participantes do estudo BALANCE Program. Brasil, 2013-2015.

\begin{tabular}{|c|c|c|c|c|}
\hline \multirow[t]{2}{*}{ Características } & \multicolumn{4}{|c|}{ IQD-R } \\
\hline & $\begin{array}{c}\text { Tercil } 1 \\
\text { (até } 47,7 \text { ) }\end{array}$ & $\begin{array}{c}\text { Tercil } 2 \\
\text { (de } 47,8 \text { a } 58,0 \text { ) }\end{array}$ & $\begin{array}{c}\text { Tercil } 3 \\
(58,1 \text { ou mais })\end{array}$ & Valor de $p$ \\
\hline Idade (média \pm DP) & $62,4 \pm 8,2(n=387)$ & $63,2 \pm 8,0(n=386)$ & $63,2 \pm 7,7(n=387)$ & 0,259 * \\
\hline \multicolumn{5}{|l|}{$\operatorname{Sexo}(n, \%)$} \\
\hline Feminino & $150(38,8)$ & $154(39,9)$ & $165(42,6)$ & 0,528 \\
\hline Masculino & $237(61,2)$ & $232(60,1)$ & $222(57,4)$ & \\
\hline \multicolumn{5}{|l|}{ Classe socioeconômica (n, \%) } \\
\hline A & $16(4,2)$ & $10(2,6)$ & $15(4,0)$ & 0,172 \\
\hline B & $101(26,6)$ & $119(31,5)$ & $106(28,0)$ & \\
\hline $\mathrm{C}$ & $214(56,5)$ & $207(54,8)$ & $229(60,4)$ & \\
\hline $\mathrm{D}$ e $\mathrm{E}$ & $48(12,7)$ & $42(11,1)$ & $29(7,7)$ & \\
\hline \multicolumn{5}{|l|}{ Escolaridade (n, \%) } \\
\hline Analfabeto/Fundamental incompleto & $105(27,5)$ & $99(26,4)$ & $86(22,8)$ & 0,483 \\
\hline Ensino Fundamental 1 & $131(34,3)$ & $118(31,5)$ & $130(34,4)$ & \\
\hline Ensino Fundamental 2 & $55(14,4)$ & $46(12,3)$ & $46(12,2)$ & \\
\hline Ensino Médio & $62(16,2)$ & $77(20,5)$ & $76(20,1)$ & \\
\hline Ensino Superior & $29(7,6)$ & $35(9,3)$ & $40(10,6)$ & \\
\hline \multicolumn{5}{|l|}{ Região de moradia no Brasil (n, \%) } \\
\hline Nordeste & $96(24,8)$ & $89(23,1)$ & $87(22,5)$ & 0,347 \\
\hline Norte & $20(5,2)$ & $24(6,2)$ & $20(5,2)$ & \\
\hline Centro-oeste & $27(7,0)$ & $27(7,0)$ & $13(3,4)$ & \\
\hline Sudeste & $144(37,2)$ & $144(37,3)$ & $148(38,2)$ & \\
\hline Sul & $100(25,8)$ & $102(26,4)$ & $119(30,7)$ & \\
\hline \multicolumn{5}{|l|}{$\operatorname{DCV}(\mathrm{n}, \%)$} \\
\hline Doença arterial periférica & $27(7,0)$ & $38(9,8)$ & $41(10,6)$ & 0,183 \\
\hline Doença arterial coronariana & $364(94,1)$ & $361(93,5)$ & $363(93,8)$ & 0,954 \\
\hline Doença cerebrovascular & $42(10,9)$ & $34(8,8)$ & $34(8,8)$ & 0,530 \\
\hline Custo direto (média \pm DP) & $3.9 \pm 2.3(n=387)$ & $3.9 \pm 2.0(n=386)$ & $3.8 \pm 1.8(n=387)$ & $0,680 * \star$ \\
\hline
\end{tabular}

DCV: doença cardiovascular; DP: desvio padrão.

Nota: dados referentes ao início do programa. Valor de p: teste qui-quadrado.

* Comparação das médias por meio da análise de variância (ANOVA);

** Comparação das médias pelo modelo linear generalizado com distribuição gama.

países, dadas as diferenças na composição da alimentação e variabilidade de preços em níveis local e internacional, incluindo nível de produtividade, salários e poder de compra, taxas de câmbio e inflação, impostos e/ou subsídios, sazonalidade, importação de alimentos, entre outros fatores 16.

Em relação à qualidade nutricional da dieta, é importante mencionar que o método selecionado para a avaliação da alimentação influencia significativamente os resultados, pois é possível compor indicadores de qualidade nutricional baseados em métodos, componentes e características bastante distintas, a depender do aspecto a ser considerado 17.

A utilização de índices sintéticos de qualidade nutricional da alimentação baseados em várias dimensões de interesse em termos de desfechos em saúde é uma entre as várias formas de avaliar a qualidade da dieta. Tais índices refletem a adequação da dieta do indivíduo e/ou da população segundo uma recomendação nutricional estabelecida por diretrizes ou guias, que devem ser adaptados em nível local para contemplar características socioculturais, econômicas e climáticas específicas. Considerando-se a enorme variedade de alimentos, preparações e nutrientes, os índices sintéticos 


\section{Figura 1}

Correlação entre escores do Índice de Qualidade da Dieta Revisado (IQD-R) e custo direto do dia alimentar de indivíduos com doença cardiovascular, participantes do estudo BALANCE Program, segundo tercis do IQD-R. Brasil, 2013-2015.

1a) Todos os recordatórios de 24 horas

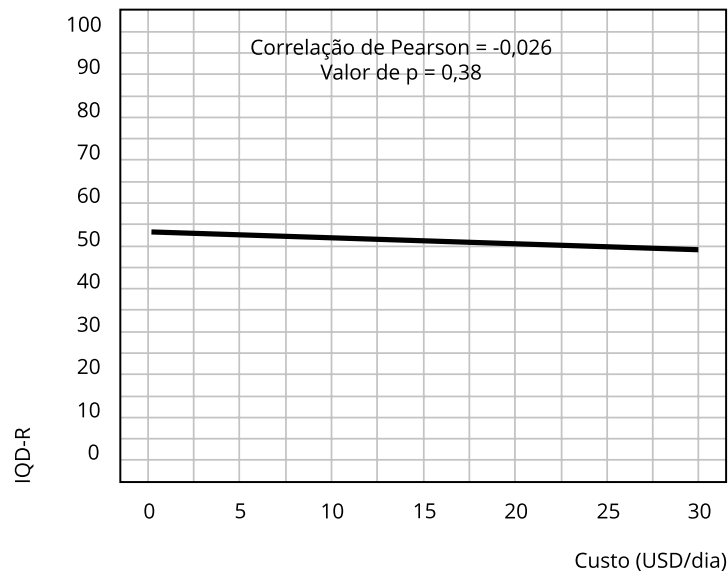

1c) Adesão - 2º tercil

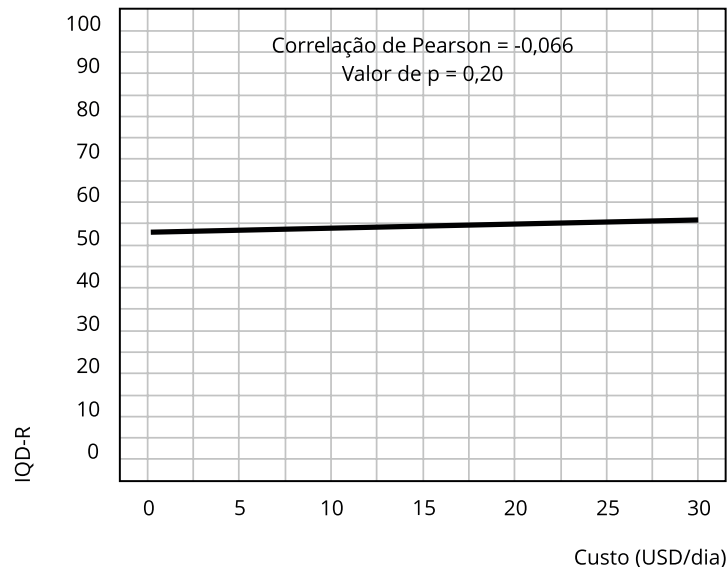

1b) Adesão - 1ํ tercil

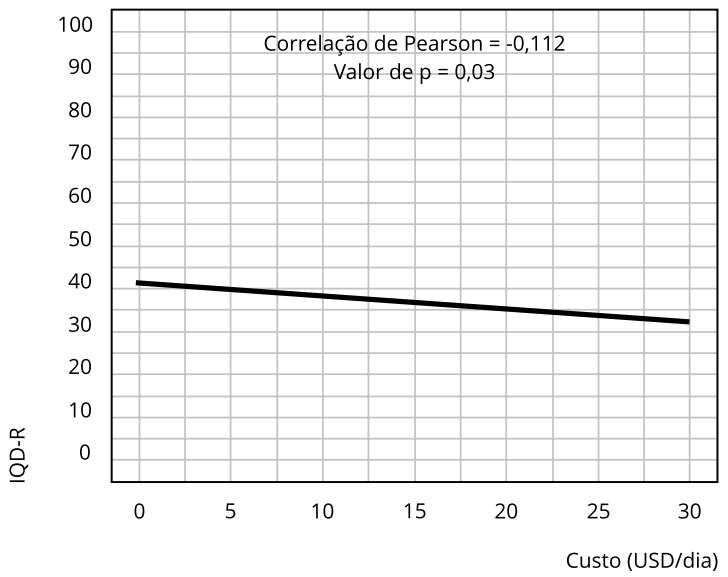

1d) Adesão - 3o tercil

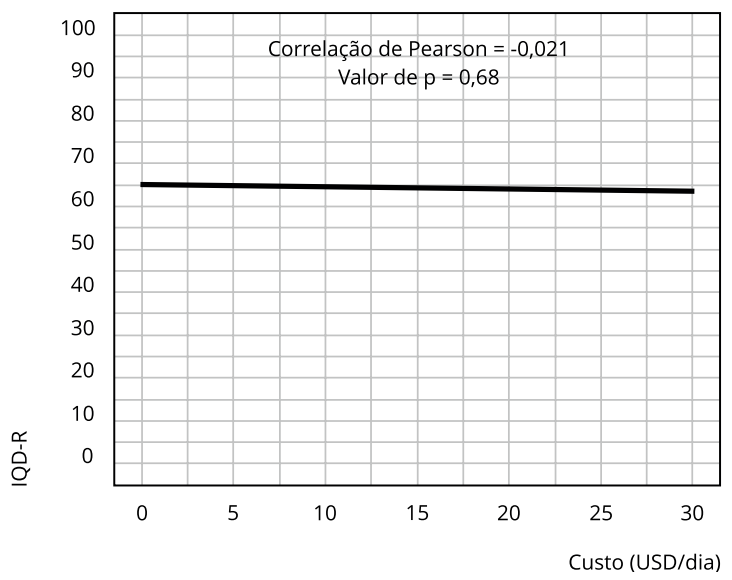

constituem uma medida resumo para refletir o padrão alimentar do indivíduo e/ou da população a ser avaliada. O índice escolhido no presente estudo foi o IQD-R validado para a aplicação na população brasileira, que constitui uma adaptação do HEI-2005, elaborado para a população norte-americana 17.

A adesão à qualidade da dieta não foi melhor em nenhuma das categorias exploradas (idade, sexo, escolaridade, classe socioeconômica, região de moradia e DCV prévia). Diferentemente do encontrado em outros estudos que identificaram qualidade alimentar melhor entre mulheres, indivíduos maior renda e escolaridade 18,19 .

Em relação ao custo, estudos que avaliaram o custo direto e a qualidade nutricional da alimentação com base no HEI-2005 já foram conduzidos anteriormente, sendo que uma meta-análise não identificou diferenças nos custos das dietas consideradas mais e menos saudáveis (USD 1,61 PPP por dia por pessoa, IC95\%: -0,61; 3,84) 20. Carlson e colaboradores também não confirmaram correlação 
entre os escores de adesão à qualidade da dieta e os custos diretos 21 . Tais resultados corroboram as evidências do presente artigo, podendo ser usados como argumento para incentivo à adesão a uma alimentação saudável.

Por outro lado, a mesma meta-análise citada anteriormente identificou diferenças de custos pela avaliação da qualidade da alimentação segundo padrões da dieta mediterrânea, que tornaram a alimentação diária mais cara (USD 1,18 PPP por dia por pessoa, IC95\%: 0,01; 2,36) 20. Mais recentemente, um estudo espanhol realizado com a população jovem verificou que, quanto maior a adesão à dieta mediterrânea, maior o gasto com a alimentação entre indivíduos mais jovens 22 . No caso, o custo direto da alimentação diária foi EUR 0,72 superior no nível de maior adesão ao escore de qualidade nutricional. Os indivíduos mais aderentes à dieta mediterrânea dispenderam maiores valores com o consumo de peixes, leite e derivados, frutas e vegetais.

Apesar das evidências clínicas quanto ao efeito protetor para DCV decorrentes do consumo de uma dieta mediterrânea 7 , não foram identificados estudos que relacionam o custo direto e a qualidade nutricional desta dieta em nível populacional no Brasil.

O presente artigo constitui o primeiro estudo que avaliou o custo direto da alimentação de indivíduos adultos brasileiros com DCV, utilizando estimativa de custos diários obtidos com base no relato individual do consumo alimentar em associação com preços médios de mercado coletados em supermercados das cinco regiões brasileiras.

O custo direto médio com alimentação foi de USD 3,89 por dia, valor superior ao identificado em um estudo baseado em dados de pesquisa transversal de representatividade populacional conduzido pelo IBGE, que estimou o custo da alimentação de indivíduos adultos brasileiros em torno de USD 2,16 por dia por pessoa ${ }^{10}$. Destacam-se diferenças substanciais na metodologia usada para a estimativa de custos da alimentação e no delineamento do estudo, assim como heterogeneidade em termos de grupo populacional em investigação de ambos os estudos.

Enquanto o estudo de Verly Jr. et al. 10 utilizou dados do consumo alimentar individual em uma subamostra de domicílios da Pesquisa de Orçamentos Familiares de 2008-2009 (POF), para a estimativa do custo direto da alimentação com base em preços dos alimentos extraídos de dados do gasto domiciliar com alimentação relatado pela família em uma semana, o presente trabalho usou dados de indivíduos em uma coorte de 36 meses sob acompanhamento por evento cardiovascular. Adicionalmente, os estudos baseiam-se em preços de mercado coletados em períodos distintos da economia brasileira (período de crescimento econômico em 2008-2009 em comparação com o período de estagnação econômica entre 2012-2018) e convertidos em dólares com base em taxas de câmbio de diferentes datas no contexto de uma economia volátil.

Em relação ao método de estimativa dos custos diretos com alimentação, há discussão acerca da obtenção dos dados de consumo alimentar por meio de questionários de frequência alimentar (em lugar de R24h) e uso de preços de mercado coletados diretamente em equipamentos de varejo locais (por exemplo, supermercados e outros), que seriam mais representativos da realidade populacional do que outras técnicas de estimativa de custos baseadas em gastos relatados pelos indivíduos ou famílias em um domicílio 23 .

O estudo avaliou a qualidade da dieta pelo IQD-R, que para esta função incorporou recomendações nutricionais internacionais e nacionais vigentes no momento de elaboração da ferramenta 14 . No entanto, atualmente, a principal diretriz nacional para a orientação alimentar da população é o Guia Alimentar para a População Brasileira 24, que recomenda o consumo de alimentos segundo o grau de processamento, natureza e finalidade (alimentos: in natura, minimamente processados, processados e ultraprocessados) 25. No Brasil, a participação calórica dos alimentos ultraprocessados na alimentação diária é cerca de 2 vezes menor do que no Reino Unido e também é mais caro quando comparado aos demais alimentos 11,26. Nessa direção, no presente estudo, o custo médio no tercil mais alto de adesão ao IQD-R foi ligeiramente mais barato, apesar de não significativo.

Em conclusão, esta investigação fornece subsídios para o estímulo ao consumo de alimentos saudáveis em termos de melhoria da qualidade da dieta de indivíduos adultos de baixa renda em um país em desenvolvimento, tendo em vista a baixa influência no custo diário com alimentação, baseando-se na identificação de dietas com diferentes níveis de qualidade nutricional a preços semelhantes nas cinco regiões brasileiras. 


\section{Colaboradores}

C. R. Torreglosa participou do planejamento, desenho, análise, escrita e revisão. F. M. Sarti participou do desenho, escrita e revisão do artigo. A. C. Bersch-Ferreira participou da coleta de dados, análise e revisão. B. Weber participou do planejamento, desenho e revisão do artigo. R. H. N. Santos participou do planejamento, análise e revisão. A. D. P. Chiavegatto Filho participou do planejamento, desenho, análise e revisão.

\section{Informações adicionais}

ORCID: Camila Ragne Torreglosa (0000-00029385-0148); Flávia Mori Sarti (0000-0003-28342005); Ângela Cristine Bersch-Ferreira (00000003-3478-781X); Bernardete Weber (0000-00031912-652X); Renato Hideo Nakagawa Santos (0000-0002-0650-212X); Alexandre D. P. Chiavegatto Filho (0000-0003-3251-9600).

\section{Agradecimentos}

Agradecemos a Messilyana de Oliveira Mesquita, Olivia Farias dos Santos, Amanda de Oliveira Guimarães e Arnaldo Torreglosa, que trabalharam na coleta de dados dos preços. E a todos os participantes do estudo BALANCE Program.

\section{Referências}

1. Joseph P, Leong D, McKee M, Anand SS, Schwalm J-D, Teo K, et al. Reducing the global burden of cardiovascular disease, part 1. Circ Res 2017; 121:677-94.

2. Ribeiro ALP, Duncan BB, Brant LCC, Lotufo PA, Mill JG, Barreto SM. Cardiovascular health in Brazil. Circulation 2016; 133:422-33.

3. World Health Organization. Health promotion: bridging the equity gap. In: World Health Organization, editor. Fifth Global Conference on Health Promotion: bridging the equity gap. Mexico City: World Health Organziation; 2000. p. 1-49.

4. Davis C, McMurry K, McMurry M. American Heart Association 2010 Dietary Guidelines. http://americanheart.biz/idc/groups/ heart-public/@wcm/@adv/documents/down loadable/ucm_312853.pdf (acessado em 01/ Mar/2018).

5. Kromhout D, Menotti A, Kesteloot H, Sans S. Prevention of coronary heart disease by diet and lifestyle evidence from prospective crosscultural, cohort, and intervention studies. Circulation 2002; 105:893-8.

6. Piepoli MF, Hoes AW, Agewall S, Albus C, Brotons C, Catapano AL, et al. 2016 European guidelines on cardiovascular disease prevention in clinical practice. Eur Heart J 2016; 37:2315-81.

7. Estruch R, Ros E, Salas-Salvadó J, Covas M-I, Corella D, Arós F, et al. Primary prevention of cardiovascular disease with a Mediterranean diet. N Engl J Med 2013; 368:1279-90.

8. Glanz K, Basil M, Maibach E, Goldberg J, Snyder D. Why Americans eat what they do: taste, nutrition, cost, convenience, and weight control concerns as influences on food consumption. J Am Diet Assoc 1998; 98:1118-26.

9. Darmon N, Drewnowski A. Contribution of food prices and diet cost to socioeconomic disparities in diet quality and health: a systematic review and analysis. Nutr Rev 2015; 73:643-60.

10. Verly-Jr E, Sichieri R, Darmon N, Maillot M, Sarti FM. Planning dietary improvements without additional costs for low-income individuals in Brazil: linear programming optimization as a tool for public policy in nutrition and health. Nutr J 2019; 18:40.

11. Claro RM, Maia EG, Costa BVL, Diniz DP. Preço dos alimentos no Brasil: prefira preparações culinárias a alimentos ultraprocessados. Cad Saúde Pública 2016; 32:e0104715.

12. Weber B, Bersch-Ferreira AC, Torreglosa CR, Marcadenti A, Lara ES, Silva JT, et al. Implementation of a Brazilian cardioprotective nutritional (BALANCE) program for improvement on quality of diet and secondary prevention of cardiovascular events: a randomized, multicentre trial. Am Heart J 2019; 215:187-97. 
13. Benzecry EH, Pinheiro ABV, Lacerda EMA, Gomes MCS, Costa V. Tabela para avaliação de consumo alimentar em medidas caseira. Rio de Janeiro: Editora Atheneu; 2008.

14. Previdelli ÀN, Andrade SC, Pires MM, Ferreira SRG, Fisberg RM, Marchioni DM. Índice de Qualidade da Dieta Revisado para população brasileira. Rev Saúde Pública 2011; 45:794-8.

15. Guenther PM, Reedy J, Krebs-Smith SM. Development of the Healthy Eating Index-2005. J Am Diet Assoc 2008; 108:1896-901.

16. Lee A, Mhurchu CN, Sacks G, Swinburn B, Snowdon W, Vandevijvere S, et al. Monitoring the price and affordability of foods and diets globally. Obes Rev 2013; 14 Suppl 1:82-95.

17. Liese AD, Krebs-Smith SM, Subar AF, George SM, Harmon BE, Neuhouser ML, et al. The dietary patterns methods project: synthesis of findings across cohorts and relevance to dietary guidance. J Nutr 2015; 145:393-402.

18. Pinto D, Fernandes S, Sônia M, Duarte L, Pessoa CS, Ribeiro AQ. Evaluation of diet quality of the elderly and associated factors. Arch Gerontol Geriatr 2017; 72:174-80.

19. Moreira PRS, Rocha NP, Milagres LC, de Novaes JF. Critical analysis of the diet quality of the Brazilian population according to the Healthy Eating Index: a systematic review. Ciênc Saúde Colet 2015; 20:3907-23.

20. Rao M, Afshin A, Singh G, Mozaffarian D. Do healthier foods and diet patterns cost more than less healthy options? A systematic review and meta-analysis. BMJ Open 2013; 3:e004277.
21. Carlson A, Dong D, Lino M. Association between total diet cost and diet quality is limited. J Agric Resour Econ 2014; 39:47-68.

22. Schröder H, Gomez SF, Ribas-Barba L, PérezRodrigo C, Bawaked RA, Fíto M, et al. Monetary diet cost, diet quality, and parental socioeconomic status in Spanish youth. PLoS One 2016; 11:e0161422.

23. Monsivais P, Perrigue MM, Adams SL, Drewnowski A. Measuring diet cost at the individual level: a comparison of three methods. Eur J Clin Nutr 2013; 67:1220-5.

24. Departamento de Atenção Básica, Secretaria de Atenção Básica, Ministério da Saúde. Guia alimentar para a população brasileira. 2a Ed. Brasília: Ministério da Saúde; 2014.

25. Monteiro CA, Levy RB. A new classification of foods based on the extent and purpose of their processing. Cad Saúde Pública 2010; 26:2039-49.

26. Moubarac JC, Claro RM, Baraldi LG, Levy RB, Martins APB, Cannon G, et al. International differences in cost and consumption of readyto-consume food and drink products: United Kingdom and Brazil, 2008-2009. Glob Public Health 2013; 8:845-56. 


\section{Abstract}

Adherence to a healthy diet depends on factors such as food prices, while studies in developed countries have identified higher costs of more nutritional foods. The current study aimed to assess the direct food expenditures by adults with cardiovascular disease in Brazil, investigating the relationship between cost and quality of diet. The study used data from a randomized clinical trial, the BALANCE Program. The current study is a cross-sectional baseline analysis of participants with high adherence to the trial, conducted in 35 sites in all five major geographic regions of $\mathrm{Bra}$ zil. Food consumption by 1,160 individuals was collected with a 24-hour dietary recall (24HR), quality of diet was measured with the Diet Quality Index Revised (DQI-R), and direct food costs were estimated from market prices. No significant differences were observed between tertiles of adherence in the direct costs of food or individual characteristics. When all the 24HR were analyzed, there was no correlation between cost and quality of diet $(r=0.38 ; p=0.17)$, while analysis by tertiles showed a weak correlation in the lowest tertile of adherence $(r=-0.112 ; p=0.03)$. The study showed absence of differences between direct costs of healthy versus unhealthy foods, a finding that can serve as an incentive for adherence to food recommendations in Brazil, thereby minimizing barriers to the adoption of healthy lifestyles.

Secondary Prevention; Diet; Cost and Cost Analysis

\section{Resumen}

La adhesión a una alimentación saludable depende de factores como los precios de los alimentos, siendo que algunos estudios realizados en paises desarrollados apuntan hacia un mayor coste de una alimentación de mejor calidad nutricional. El objetivo del presente estudio fue evaluar el coste directo de la alimentación en individuos adultos con enfermedad cardiovascular en Brasil, investigando la relación entre el coste y la calidad de la dieta. Se utilizaron los datos de un ensayo clínico aleatorio, el BALANCE Program. La investigación actual es un análisis transversal desde el principio con participantes de alta adhesión al estudio, realizado en 35 centros de las cinco regiones brasileñas. El consumo alimentario de la muestra con 1.160 individuos fue recogido mediante el recordatorio alimentario de 24 horas (R24h), la evaluación de la calidad de la dieta se evaluó mediante el Índice de Calidad de la Dieta Revisado (IQD-R por sus siglas en portugués) y los costes directos de la alimentación se estimaron mediante precios de mercado. No se observaron diferencias significativas en el coste directo de la alimentación o características de los individuos entre los terciles de adhesión. Cuando se analizaron todos los recordatorios no hubo correlación entre coste y calidad de la dieta $(r=0,38 ; p=0,17)$, ya en el análisis por terciles hubo una débil correlación entre el menor tercil de adhesión $(r=-0,112 ; p=0,03)$. El presente estudio apuntó una ausencia de diferencias entre los costes directos de la alimentación clasificada como saludable y aquella con peor calidad nutricional, lo que puede ser un incentivo para la adhesión a las orientaciones alimentarias en Brasil, minimizando barreras para la adopción de estilos de vida saludables.

Dieta; Prevención Secundária; Costos y Análisis de Costos
Recebido em 23/Nov/2019

Versão final reapresentada em 09/Mar/2020

Aprovado em 31/Mar/2020 